\title{
Hölder continuity of solutions to the Dirichlet problem for SPDEs with spatially correlated noise*
}

\author{
Ruoyang $\mathrm{Liu}^{\dagger} \quad$ Kai $\mathrm{Du}^{\ddagger}$
}

\begin{abstract}
This paper obtains Hölder continuity of the mild solution to a stochastic parabolic equation defined on a bounded domain with homogeneous Dirichlet boundary condition. The Gaussian noise involved in the equation is white in time and correlated in space. The approach is based on a new Hölder-type estimate for the Green function in the domain.
\end{abstract}

Keywords: Stochastic partial differential equations; Dirichlet problem; Hölder continuity; correlated noise.

MSC2020 subject classifications: $60 \mathrm{H} 15 ; 35 \mathrm{R} 60$.

Submitted to ECP on January 27, 2021, final version accepted on October 16, 2021.

\section{Introduction}

This paper concerns the Dirichlet problem for the following stochastic partial differential equation (SPDE) in a bounded domain $D \subset \mathbb{R}^{d}$ :

$$
\begin{aligned}
\partial_{t} u(t, x) & =\mathcal{L} u(t, x)+g(t, x, u)+\sigma(t, x, u) \dot{W}, & & t \geq 0, x \in D, \\
u(t, x) & =0, & & t \geq 0, x \in \partial D, \\
u(0, x) & =\psi(x), & & x \in D,
\end{aligned}
$$

where $\mathcal{L}$ is a second-order differential operator defined as

$$
\mathcal{L} u(t, x):=a_{i j}(t, x) \partial_{i j} u(t, x)+b_{i}(t, x) \partial_{i} u(t, x),
$$

and $\dot{W}$ is the centered Gaussian noise that satisfies

$$
\mathbb{E}[\dot{W}(t, x) \dot{W}(s, y)]=\delta_{0}(t-s) f(x-y),
$$

where $\delta_{0}(\cdot)$ denotes the Dirac measure and $f$ is a nonnegative and continuous function defined on $\mathbb{R}^{d} \backslash\{0\}$. Einstein summation convention is used in this paper.

*The research of Kai $\mathrm{Du}$ was partially supported by National Key R\&D Program of China (No. 2018YFA0703900), by National Natural Science Foundation of China (No. 11801084), and by Natural Science Foundation of Shanghai (No. 20ZR1403600).

${ }^{\dagger}$ School of Mathematical Sciences, Fudan University, Shanghai 200433, China. E-mail: 18110180044@fudan. edu.cn

${ }^{\ddagger}$ Shanghai Center for Mathematical Sciences, Fudan University, Shanghai 200438, China. E-mail: kdu@ fudan.edu.cn. 
SPDEs with space-time noise have been widely studied. Although stochastic integrals with respect to space-time noise are well-defined in very general settings (cf. [27, 4, $20,3]$ ), the analytic property of the solution to an SPDE is affected significantly by the specific form of the noise, and in some cases, the solution may not be a function but only a distribution in Schwartz's sense. For example, if the noise is white in space and time, only in one-dimensional case the solutions to second-order SPDEs are functionvalued (cf. [27, 13]). On the other hand, when considering nonlinear SPDEs, the solutions are expected to be regular so that the nonlinear mappings can be defined. For one-dimensional case, many results have been done for both Cauchy problems and initial-boundary value problems $[27,10,11,6]$.

In the study of nonlinear SPDEs, Dalang and Frangos [5] and Dalang [4] proposed the following condition on the space-time noise: the spectral measure $\mu_{f}$, defined as the inverse Fourier transform of $f$ in (1.3), satisfies

$$
\int_{\mathbb{R}^{d}} \frac{\mu_{f}(d \xi)}{1+|\xi|^{2}}<\infty
$$

Under this condition, Dalang [4] proved that the Cauchy problem for SPDE (1.1) has a unique mild solution that satisfies $\sup _{t, x} \mathbb{E}\left[|u(t, x)|^{p}\right]<\infty$ for any $p \geq 1$, but did not show whether the solution has a continuous modification. Later, Sanz-Solé and Sarrà [24, 23] proposed a reinforcing condition:

$$
\int_{\mathbb{R}^{d}} \frac{\mu_{f}(d \xi)}{\left(1+|\xi|^{2}\right)^{1-\eta}}<\infty \quad \text { with } \eta \in(0,1)
$$

under which they proved Hölder continuity of solutions to the Cauchy problem for $\operatorname{SPDE}$ (1.1) with $\mathcal{L}=\Delta$. Their result was extended by Márquez-Carreras and Sarrà [19] to the Dirichlet problem with $D=[0,1]^{d}$ and $\mathcal{L}=\Delta$. It is worth noting that condition (1.4) alone cannot guarantee the existence of a continuous version of the solution (cf. $[2,1]$ ).

Krylov et.al. established a complete $L_{p}$-theory on the whole space [16] and domain $[15,14]$. By Sobolev's embedding, the regularity in proper Hölder spaces can be obtained, but requires higher regularities of the data.

Most literatures about SPDEs on bounded domains in the sense of Walsh are dealing with convex domains (c.f. [12, 25]). In the last decade, the interests on the results including large deviation principle, intermittency and spatial asymptotics of the solutions have grown up considerably. However, all of these results deal with the special domains such as $\mathbb{R}^{d},[0,1]$ or a closed ball (c.f. $[19,22,7,26]$ ). Since the shape of the domains play a crucial role, some results do not extend directly to general bounded domains (c.f. [21]). This article allows to go a step further on the assumptions of domains.

The aim of this paper is to prove Hölder continuity of solutions to SPDE (1.1) in general bounded domains, under condition (1.5). The approach in [19] is based on the specific form of the heat kernel on $[0,1]^{d}$, which is constructed by the method of images [8]. Their approach does not seem to work for $\mathcal{L}$ in (1.2) and general domains. Our method is based on a crucial estimate of the Green function associated with $\mathcal{L}$ (see Lemma 3.3). We prove that the mild solution is Hölder continuous with exponents $\eta / 2-\varepsilon$ in time and $\eta-\varepsilon$ in space for any $\varepsilon>0$. The indices coincide with those for the Cauchy problem (cf. [23]) and the Dirichlet problem with $D=[0,1]^{d}$ (cf. [19]).

We remark that some other assumptions instead of condition (1.5) were proposed in the literature to study the continuity of solutions to SPDEs. For example, Sanz-Solé and Vuillermot [25] introduced the condition

$$
\sup _{(t, x) \in[0, T] \times D} \int_{0}^{t}(t-\tau)^{-\eta} \sum_{j=1}^{\infty} \lambda_{j}\left(\int_{D}|G(t, x ; \tau, y)| e_{j}(y) d y\right)^{2} d \tau<\infty
$$


involving the Green function for $\mathcal{L}$, and obtained Hölder regularity for SPDE (1.1) in bounded convex domains. As condition (1.6) is weaker than condition (1.5), it is interesting to extend our results under it, especially for non-convex domains.

This paper is organized as follows. Section 2 gives the main theorem after introducing notations and assumptions. In Section 3, we apply Kolmogorov's continuity criterion and crucial esitmates of the Green function to prove Hölder continuity of the solution to SPDE (1.1).

\section{Notation and main results}

Let $\mathbb{R}^{d}$ be the Euclidean space of points $x=\left(x^{1}, \ldots, x^{d}\right)$ and $B_{r}(x):=\left\{y \in \mathbb{R}^{d}\right.$ : $|x-y|<r\}$. Let $D \subset \mathbb{R}^{d}$ be a bounded domain. $C^{m+\beta}(D)$ denotes the usual Hölder space with $m=0,1, \ldots$ and $\beta \in(0,1)$ (cf. [18]); $C_{0}(D)$ consists of all continuous real-valued functions vanishing on $\partial D$. Let $T>0$ be a fixed constant. Define $Q_{T}:=[0, T] \times D$.

For $i=1, \ldots, d$, we write $\partial_{i} u=\partial u / \partial x^{i}$ and $\nabla u=\left(\partial_{1} u, \ldots, \partial_{d} u\right)$.

Let $\left(\Omega, \mathcal{F},\left\{\mathcal{F}_{t}\right\}, \mathbf{P}\right)$ be a complete filtered probability space and $\mathcal{P}$ be the predictable $\sigma$-algebra generated by $\left\{\mathcal{F}_{t}\right\}$. Let $W=\left\{W(\varphi), \varphi \in \mathcal{D}\left(\mathbb{R}^{d+1}\right)\right\}$ be a centered Gaussian random field with covariance functional given by

$$
J(\varphi, \psi):=\int_{\mathbb{R}_{+}} \int_{\mathbb{R}^{d}} \int_{\mathbb{R}^{d}} \varphi(s, x) f(x-y) \psi(s, y) d x d y d s,
$$

where $f: \mathbb{R}^{d} \backslash\{0\} \rightarrow \mathbb{R}_{+}$is a continuous function with $f(x)=f(-x)$ for all $x$. We assume that the inverse Fourier transform of $f$ is a non-negative tempered measure on $\mathbb{R}^{d}$, which is denoted by $\mu_{f}$, and satisfies (1.5).

Fix a constant $\beta \in(0,1)$. We assume the boundary $\partial D \in C^{1+\beta}$, the definition of which is standard (cf. [18, Page 9]).

Definition 2.1 (Boundary regularity). We write $\partial D \in C^{1+\beta}$ if there is a constant $\rho_{0}>0$ such that $\partial D \cap B_{\rho_{0}}(x)$ for each $x \in \partial D$ is a connected surface whose equation under a local coordinate system $\left\{y^{i}\right\}$ is given by $y^{1}=\Psi\left(y^{2}, \ldots, y^{d}\right)$ with $\Psi \in C^{1+\beta}$.

Define the Green function $G(t, x ; s, y)$ associated with $\mathcal{L}$ on $D$ as follows:

$$
\begin{aligned}
\frac{\partial G(t, x ; s, y)}{\partial t} & =\mathcal{L}_{x} G(t, x ; s, y), \\
\left.G(t, x ; s, y)\right|_{t=s} & =\delta_{0}(x-y), \\
\left.G(t, x ; s, y)\right|_{x \in \partial D} & =0 .
\end{aligned}
$$

Then we can introduce the mild solution to SPDE (1.1) (cf. [27]).

Definition 2.2. $A \mathcal{P} \otimes \mathcal{B}(D)$-measurable function $u(\cdot, \cdot)$ is a mild solution to SPDE (1.1) if it holds for all $(t, x) \in Q_{T}$ that

$$
\begin{aligned}
u(t, x)= & \int_{D} G(t, x ; 0, y) \psi(y) d y+\int_{0}^{t} \int_{D} G(t, x ; s, y) g(s, y, u(s, y)) d y d s \\
& +\int_{0}^{t} \int_{D} G(t, x ; s, y) \sigma(s, y, u(s, y)) W(d s, d y) .
\end{aligned}
$$

Let $\bar{D}$ be the closure of $D$.

Assumption 2.3 (Parabolicity). There exist positive constants $\lambda_{1}$ and $\lambda_{2}$ such that for any $(t, x) \in[0, T] \times \bar{D}$ and $\xi=\left(\xi^{1}, \ldots, \xi^{d}\right) \in \mathbb{R}^{d}$,

$$
\lambda_{1}|\xi|^{2} \leq a_{i j}(t, x) \xi^{i} \xi^{j} \leq \lambda_{2}|\xi|^{2} .
$$


Assumption 2.4. There exist a positive constant $A$ and a constant $\alpha \in(0,1)$ such that for all $t, s \in[0, T]$ and $x, y \in \bar{D}$,

$$
\begin{aligned}
\left|a_{i j}(t, x)-a_{i j}(s, y)\right| & \leq A\left(|x-y|^{\alpha}+|t-s|^{\frac{\alpha}{2}}\right), \\
\left|b_{i}(t, x)-b_{i}(t, y)\right| & \leq A|x-y|^{\alpha} .
\end{aligned}
$$

Assumption 2.5. The functions $g$ and $\sigma$ are $\mathcal{P} \otimes \mathcal{B}(\bar{D}) \otimes \mathcal{B}(\mathbb{R})$-measurable on $\Omega \times[0, T] \times$ $\bar{D} \times \mathbb{R}$, and there exists a constant $M$ such that

$$
\sup _{(\omega, t, x) \in \Omega \times[0, T] \times \bar{D}}[|g(\omega, t, x, 0)|+|\sigma(\omega, t, x, 0)|] \leq M .
$$

Assumption 2.6. There exists a constant $K$ such that

$$
\left|g(\omega, t, x, r)-g\left(\omega, t, x, r^{\prime}\right)\right|+\left|\sigma(\omega, t, x, r)-\sigma\left(\omega, t, x, r^{\prime}\right)\right| \leq K\left|r-r^{\prime}\right|,
$$

for any $\left(\omega, t, x, r, r^{\prime}\right) \in \Omega \times[0, T] \times \bar{D} \times \mathbb{R}^{2}$.

In view of [7, Theorem 7.2] and [25, Theorem 5], the following existence and uniqueness result follows immediately from Gaussian estimates (see Lemma 2.10).

Lemma 2.7. Let (1.4) and Assumptions 2.3-2.6 be satisfied. If $\psi \in C_{0}(D)$, then SPDE (1.1) has a unique mild solution $u$ which satisfies

$$
\sup _{(t, x) \in Q_{T}} \mathbb{E}\left[|u(t, x)|^{p}\right]<\infty, \quad \forall p \geq 2 .
$$

Our main result stated below shows that the unique mild solution of (1.1) has a Hölder continuous version.

Theorem 2.8. Let (1.5) and Assumptions 2.3-2.6 be satisfied. If $\psi \in C^{2}(D) \cap C_{0}(D)$, then the mild solution of SPDE (1.1) has a modification in the space $C^{\frac{\eta}{2}-, \eta-}([0, T] \times \bar{D}):=$ $\cap_{\varepsilon>0} C^{\frac{\eta}{2}-\varepsilon, \eta-\varepsilon}([0, T] \times \bar{D})$.

Remark 2.9. Although [7, Theorem 7.2] and [25, Theorem 5] do not address the same problem in this article, their methods can be extended based on Lemma 2.10. Besides, it has been shown that $\dot{W}$ in our paper satisfies their assumptions on the noise (see [4, Remark 10] and [25, Page 839]).

The proof of Theorem 2.8 is given in the next section. Here we remark that the property (2.2) along with Assumptions 2.5 and 2.6 ensures that the stochastic integral in $(2.1)$ is well-defined (cf. $[27,4])$. Indeed, one will see that

$$
\mathbb{E}\left[\left(\int_{0}^{t} \int_{D} \int_{D} G(t, x ; s, y) \sigma(s, y, u) f(y-z) \sigma(s, z, u) G(t, x ; s, z) d y d z d s\right)^{\frac{p}{2}}\right]
$$

is bounded for any $p \geq 2$.

The following estimates for the Green function, taken from [18, Chapter IV], play a key role in the proof of Theorem 2.8.

Lemma 2.10. Under Assumptions 2.3 and 2.4, there exist two positive constants $k_{1}$ and $k_{2}$ such that

$$
\begin{gathered}
G(t, x ; s, y) \leq k_{1}(t-s)^{-\frac{d}{2}} \exp \left(-k_{2} \frac{|x-y|^{2}}{t-s}\right), \\
\left|\nabla_{x} G(t, x ; s, y)\right| \leq k_{1}(t-s)^{-\frac{d+1}{2}} \exp \left(-k_{2} \frac{|x-y|^{2}}{t-s}\right), \\
\left|\partial_{t} G(t, x ; s, y)\right| \leq k_{1}(t-s)^{-\frac{d+2}{2}} \exp \left(-k_{2} \frac{|x-y|^{2}}{t-s}\right),
\end{gathered}
$$

for all $x, y \in \bar{D}$ and $0 \leq s<t \leq T$. 
Letting $\Gamma_{k}(t, x ; s, y)$ be the fundamental solution of $\partial_{t} u=\frac{1}{4 k} \Delta u$ in $\mathbb{R}^{d}$, the estimate (2.4) implies that there is a positive constant $k_{3}$ depending on $k_{1}, k_{2}$, and $d$ such that

$$
G(t, x ; s, y) \leq k_{3} \Gamma_{k_{2}}(t, x ; s, y),
$$

for all $x, y \in \bar{D}$ and $0 \leq s<t \leq T$. This is called Gaussian estimate, which can be easily proved by the maximum principle.

Remark 2.11. Lemma 2.10 is proved in [18, Chapter IV], of which the original version assumes the coefficients $a_{i j}$ and $b_{i}$ to be defined on $[0, T] \times \mathbb{R}^{d}$ and satisfy Assumptions 2.3 and 2.4. Although the coefficients in this paper are defined on $[0, T] \times \bar{D}$, they can easily be extended to the whole space $\mathbb{R}^{d}$ and still satisfy the same assumptions.

\section{Proof of Theorem 2.8}

To prove the Hölder continuity of the mild solution, we need the following three lemmas, of which the first one is a property of the path in the bounded domain $D$. Given $x, z \in D$, we define $\bar{P}_{x, z}$ as the set, which consists of all continuous paths satisfying the following two conditions:

(i) the endpoints of the path are $x$ and $z$;

(ii) each point on the path belongs to $\bar{D}$.

Lemma 3.1. For any bounded domain $D \subset \mathbb{R}^{d}$ with $C^{1+\beta}$ boundary, there exists a constant $K_{D}>1$ depending only on the shape of $D$ such that for any two points $x, z \in \bar{D}$, we can find a path in $\bar{P}_{x, z}$, and the length of this path is bounded by $K_{D}|x-z|$.

We postpone the proof of this lemma to the end of this section. Now we aim to treat the solution $u$ as a random field with parameter $(t, x)$, and apply the Kolmogorov's continuity criterion which is shown in [17, Lemma 1.4.1] to prove the continuity of solution $u$ up to a modification.

Lemma 3.2. Let $\{X(\mathbf{t})\}_{\mathbf{t} \in \mathbb{T}}$ be a stochastic process indexed by $\mathbf{t}=\left(t^{1}, t^{2}, \ldots, t^{N}\right) \in \mathbb{T}$ where $\mathbb{T}$ is a domain in $\mathbb{R}^{N}$. Suppose that there exist constants $C>0, p>0$, and $\alpha_{i}>0, i=1, \ldots, N$ with $\sum_{i=1}^{N} \alpha_{i}^{-1}<1$ such that uniformly for all $\mathbf{s}, \mathbf{t} \in \mathbb{T}$,

$$
\mathbb{E}\left[|X(\mathbf{t})-X(\mathbf{s})|^{p}\right] \leq C\left(\sum_{i=1}^{N}\left|t^{i}-s^{i}\right|^{\alpha_{i}}\right)
$$

then $X$ has a continuous modification $\bar{X}$. Moreover, define $\alpha_{0}:=d /\left(\sum_{i=1}^{N} \alpha_{i}^{-1}\right)$ and let $\bar{\alpha}_{i}, i=1, \ldots, N$ be positive constants less than $\alpha_{i}\left(\alpha_{0}-d\right) /\left(\alpha_{0} p\right), i=1, \ldots, N$ respectively, then we have

$$
\mathbb{E}\left[\left(\sup _{\mathbf{s} \neq \mathbf{t}} \frac{|X(\mathbf{t})-X(\mathbf{s})|}{\left(\sum_{i=1}^{N}\left|t^{i}-s^{i}\right|^{\bar{\alpha}_{i}}\right)}\right)^{p}\right]<\infty .
$$

The following lemma gives the crucial estimates. The idea for the proof of this lemma is derived from the proof of Theorem 7 of Chapter 1 in [9], which proves the estimates for the fundamental solution in $\mathbb{R}^{d}$.

Lemma 3.3. Under Assumptions 2.3 and 2.4, for any $\theta \in[0,1]$, we have:

(i) for all $0 \leq s<t \leq T$ and $x_{1}, x_{2}, y \in \bar{D}$,

$$
\begin{aligned}
& \left|G\left(t, x_{1} ; s, y\right)-G\left(t, x_{2} ; s, y\right)\right| \\
& \quad \leq C(t-s)^{-\frac{\theta}{2}}\left|x_{1}-x_{2}\right|^{\theta}\left(\Gamma_{k_{2} / 2}\left(t, x_{1} ; s, y\right)+\Gamma_{k_{2} / 2}\left(t, x_{2} ; s, y\right)\right)
\end{aligned}
$$

where $C$ is independent of $\theta, s, t, x_{1}, x_{2}$, and $y$. 
(ii) for all $0 \leq s<t_{2}<t_{1} \leq T$ and $x, y \in \bar{D}$,

$$
\begin{aligned}
& \left|G\left(t_{1}, x ; s, y\right)-G\left(t_{2}, x ; s, y\right)\right| \\
& \quad \leq C\left(t_{1}-t_{2}\right)^{\frac{\theta}{2}}\left(t_{2}-s\right)^{-\frac{\theta}{2}}\left(\Gamma_{k_{2} / 2}\left(t_{1}, x ; s, y\right)+\Gamma_{k_{2} / 2}\left(t_{2}, x ; s, y\right)\right)
\end{aligned}
$$

where $C$ is independent of $\theta, s, t_{1}, t_{2}, x$, and $y$. The constant $k_{2}$ is taken from Lemma 2.10.

Proof. The results for $\theta=0$ or 1 are direct consequences of Lemma 2.10. Therefore, we only consider the case $\theta \in(0,1)$. For any $x_{1}, x_{2} \in \bar{D}$ and $0 \leq s<t \leq T$, when $t-s \leq\left|x_{1}-x_{2}\right|^{2}$, the Gaussian estimate (2.4) indicates that

$$
\left|G\left(t, x_{1} ; s, y\right)\right| \leq k_{1}(t-s)^{-\frac{d}{2}} \exp \left(-k_{2} \frac{\left|x_{1}-y\right|^{2}}{t-s}\right)
$$

and

So we have

$$
\left|G\left(t, x_{2} ; s, y\right)\right| \leq k_{1}(t-s)^{-\frac{d}{2}} \exp \left(-k_{2} \frac{\left|x_{2}-y\right|^{2}}{t-s}\right)
$$

$$
\begin{aligned}
& \left|G\left(t, x_{1} ; s, y\right)-G\left(t, x_{2} ; s, y\right)\right| \\
& \leq k_{1}(t-s)^{\frac{\theta}{2}}(t-s)^{-\frac{d+\theta}{2}}\left[\exp \left(-k_{2} \frac{\left|x_{1}-y\right|^{2}}{t-s}\right)+\exp \left(-k_{2} \frac{\left|x_{2}-y\right|^{2}}{t-s}\right)\right] \\
& \leq k_{1}\left|x_{1}-x_{2}\right|^{\theta}(t-s)^{-\frac{d+\theta}{2}}\left[\exp \left(-k_{2} \frac{\left|x_{1}-y\right|^{2}}{t-s}\right)+\exp \left(-k_{2} \frac{\left|x_{2}-y\right|^{2}}{t-s}\right)\right] .
\end{aligned}
$$

Then we focus on the case $t-s>\left|x_{1}-x_{2}\right|^{2}$. Based on Lemma 3.1, there exist a constant $K_{D}$ and a path $P_{x_{1}, x_{2}} \in \bar{P}_{x_{1}, x_{2}}$, such that the length of $P_{x_{1}, x_{2}}$ is bounded by $K_{D}\left|x_{1}-x_{2}\right|$. Then we use (2.5) to obtain

$$
\begin{aligned}
\left|G\left(t, x_{1} ; s, y\right)-G\left(t, x_{2} ; s, y\right)\right| & \leq \sup _{\xi \in P_{x_{1} x_{2}}}\left|\nabla_{x} G(t, \xi ; s, y)\right| \cdot K_{D}\left|x_{1}-x_{2}\right| \\
& \leq \sup _{\xi \in P_{x_{1} x_{2}}} C(t-s)^{-\frac{d+1}{2}} \exp \left(-k_{2} \frac{|y-\xi|^{2}}{t-s}\right)\left|x_{1}-x_{2}\right| .
\end{aligned}
$$

Due to $\xi \in P_{x_{1} x_{2}}$, we have

$$
2|y-\xi|^{2} \geq\left|x_{1}-y\right|^{2}-2\left|x_{1}-\xi\right|^{2} \geq\left|x_{1}-y\right|^{2}-2 K_{D}^{2}\left|x_{1}-x_{2}\right|^{2} .
$$

Therefore, one has

$$
\begin{aligned}
& \left|G\left(t, x_{1} ; s, y\right)-G\left(t, x_{2} ; s, y\right)\right| \\
& \leq C(t-s)^{-\frac{d+1}{2}}\left|x_{1}-x_{2}\right|^{\theta}\left|x_{1}-x_{2}\right|^{1-\theta} \exp \left(k_{2} K_{D}^{2} \frac{\left|x_{1}-x_{2}\right|^{2}}{t-s}-\frac{k_{2}}{2} \frac{\left|x_{1}-y\right|^{2}}{t-s}\right) \\
& \leq C(t-s)^{-\frac{d+\theta}{2}}\left|x_{1}-x_{2}\right|^{\theta} \exp \left(-\frac{k_{2}}{2} \frac{\left|x_{1}-y\right|^{2}}{t-s}\right) .
\end{aligned}
$$

Hence, for all $0 \leq s<t \leq T$ and $x_{1}, x_{2}, y \in \bar{D}$, we have

$$
\begin{aligned}
& \left|G\left(t, x_{1} ; s, y\right)-G\left(t, x_{2} ; s, y\right)\right| \\
& \leq C(t-s)^{-\frac{d+\theta}{2}}\left|x_{1}-x_{2}\right|^{\theta}\left[\exp \left(-\frac{k_{2}}{2} \frac{\left|x_{1}-y\right|^{2}}{t-s}\right)+\exp \left(-\frac{k_{2}}{2} \frac{\left|x_{2}-y\right|^{2}}{t-s}\right)\right],
\end{aligned}
$$


which completes the proof of (i) in Lemma 3.3. As for the second estimate (3.2) in Lemma 3.3, we adopt a similar method as in the proof of (i). We first consider the case $t_{2}-s<t_{1}-t_{2}$. It is easy to see that $t_{1}-s<2\left(t_{1}-t_{2}\right)$, and we combine this estimate with the Gaussian estimate (2.4) to obtain that

$$
|G(\tau, x ; s, y)| \leq 2 k_{1}(\tau-s)^{-\frac{d+\theta}{2}}\left(t_{1}-t_{2}\right)^{\frac{\theta}{2}} \exp \left(-k_{2} \frac{|x-y|^{2}}{\tau-s}\right)
$$

for $\tau \in\left\{t_{1}, t_{2}\right\}$, then we can get that

$$
\begin{aligned}
& \left|G\left(t_{1}, x ; s, y\right)-G\left(t_{2}, x ; s, y\right)\right| \\
& \leq 2 k_{1}\left(t_{1}-t_{2}\right)^{\frac{\theta}{2}}\left[\left(t_{1}-s\right)^{-\frac{d+\theta}{2}} \exp \left(-k_{2} \frac{|x-y|^{2}}{t_{1}-s}\right)+\left(t_{2}-s\right)^{-\frac{d+\theta}{2}} \exp \left(-k_{2} \frac{|x-y|^{2}}{t_{2}-s}\right)\right] .
\end{aligned}
$$

On the other hand, if $t_{2}-s \geq t_{1}-t_{2}$, we have

$$
\left(t_{2}-s\right)^{-1}=\left(t_{1}-s\right)^{-1}+\left(t_{1}-t_{2}\right)\left(t_{1}-s\right)^{-1}\left(t_{2}-s\right)^{-1} \leq 2\left(t_{1}-s\right)^{-1} .
$$

Combining this estimate with (2.6) and applying the mean value theorem, one can get that there exists $\bar{t} \in\left[t_{2}, t_{1}\right]$ such that

$$
\begin{aligned}
\left|G\left(t_{1}, x ; s, y\right)-G\left(t_{2}, x ; s, y\right)\right| & \leq C(\bar{t}-s)^{-\frac{d+2}{2}}\left(t_{1}-t_{2}\right)^{\frac{\theta}{2}}\left(t_{1}-t_{2}\right)^{1-\frac{\theta}{2}} \exp \left(-k_{2} \frac{|x-y|^{2}}{\bar{t}-s}\right) \\
& \leq C\left(t_{2}-s\right)^{-\frac{d+\theta}{2}}\left(t_{1}-t_{2}\right)^{\frac{\theta}{2}} \exp \left(-k_{2} \frac{|x-y|^{2}}{t_{1}-s}\right) \\
& \leq C\left(t_{1}-s\right)^{-\frac{d}{2}}\left(t_{2}-s\right)^{-\frac{\theta}{2}}\left(t_{1}-t_{2}\right)^{\frac{\theta}{2}} \exp \left(-k_{2} \frac{|x-y|^{2}}{t_{1}-s}\right) .
\end{aligned}
$$

Therefore, for all $0 \leq s<t_{2}<t_{1} \leq T$ and $x, y \in \bar{D}$, we have

$$
\left|G\left(t_{1}, x ; s, y\right)-G\left(t_{2}, x ; s, y\right)\right| \leq C\left(t_{1}-t_{2}\right)^{\frac{\theta}{2}}\left(t_{2}-s\right)^{-\frac{\theta}{2}}\left(\Gamma_{k_{2} / 2}\left(t_{1}, x ; s, y\right)+\Gamma_{k_{2} / 2}\left(t_{2}, x ; s, y\right)\right)
$$

Then Lemma 3.3 is proved.

We now start to prove Theorem 2.8.

Proof of Theorem 2.8. Based on Definition 2.2, we only need to prove the Hölder continuity of each terms in the right hand side of (2.1). For non-zero $\psi \in C^{2}(D) \cap C_{0}(D)$, one can take a simple transformation to reduce the problem to the case with $\psi=0$; for example, consider the equation of $v=u-\psi$. Besides, the proof of the term consisting $g(t, x, u(t, x))$ can be easily proved with Lemma 2.7 and 3.3, Hölder inequality, Assumptions 2.5 and 2.6. Therefore, we pay more attention to the term

$$
U(t, x):=\int_{0}^{t} \int_{D} G(t, x ; s, y) \sigma(s, y, u(s, y)) W(d s, d y) .
$$

We divide the proof into two steps, which prove the continuity for space and time separately. 
Step 1: Let $x_{1}$ and $x_{2}$ be two different points in $\bar{D}$. By the Burkholder's inequality (see the proof of [4, Theorem 5]) and $f \geq 0$, for all $t \in(0, T]$ and $p \geq 2$, we have

$$
\begin{aligned}
& \mathbb{E}\left[\left|U\left(t, x_{1}\right)-U\left(t, x_{2}\right)\right|^{p}\right] \\
& \leq C \mathbb{E}\left[\left(\int_{0}^{t} \int_{D} \int_{D}\left|G\left(t, x_{1} ; s, y\right)-G\left(t, x_{2} ; s, y\right)\right| \cdot|\sigma(s, y, u(s, y))| \cdot f(y-z)\right.\right. \\
& \left.\left.\quad \cdot|\sigma(s, z, u(s, z))| \cdot\left|G\left(t, x_{1} ; s, z\right)-G\left(t, x_{2} ; s, z\right)\right| d y d z d s\right)^{\frac{p}{2}}\right] .
\end{aligned}
$$

Similar to the proof of [4, Theorem 13], we use the Hölder inequality, stochastic Fubini theorem, Assumptions 2.5 and 2.6, and Lemma 2.7 to acquire that

$$
\begin{aligned}
& \mathbb{E}\left[\left|U\left(t, x_{1}\right)-U\left(t, x_{2}\right)\right|^{p}\right] \\
& \leq C\left(1+\sup _{(s, x) \in[0, t] \times D} \mathbb{E}\left[|u(s, x)|^{p}\right]\right)\left(\int_{0}^{t} \int_{D} \int_{D}\left|G\left(t, x_{1} ; s, y\right)-G\left(t, x_{2} ; s, y\right)\right|\right. \\
& \left.\quad f(y-z) \cdot\left|G\left(t, x_{1} ; s, z\right)-G\left(t, x_{2} ; s, z\right)\right| d y d z d s\right)^{\frac{p}{2}} .
\end{aligned}
$$

Set $\theta \in(0,1)$ a constant to be determined. Combine (3.3) with (3.1) to acquire

$$
\begin{aligned}
& \mathbb{E}\left[\left|U\left(t, x_{1}\right)-U\left(t, x_{2}\right)\right|^{p}\right] \\
& \leq C\left|x_{1}-x_{2}\right|^{\theta p}\left[\int_{0}^{t}(t-s)^{-\theta} \int_{\mathbb{R}^{d}} \int_{\mathbb{R}^{d}}\left(\Gamma_{k_{2} / 2}\left(t, x_{1} ; s, y\right)+\Gamma_{k_{2} / 2}\left(t, x_{2} ; s, y\right)\right)\right. \\
& \left.\quad \cdot f(y-z) \cdot\left(\Gamma_{k_{2} / 2}\left(t, x_{1} ; s, z\right)+\Gamma_{k_{2} / 2}\left(t, x_{2} ; s, z\right)\right) d y d z d s\right]^{\frac{p}{2}} .
\end{aligned}
$$

Define

$$
F(x, \varsigma):=\int_{\mathbb{R}^{d}} \int_{\mathbb{R}^{d}} \Gamma_{k_{2} / 2}(t, x ; s, y) f(y-z) \Gamma_{k_{2} / 2}(t, \varsigma ; s, z) d y d z
$$

for any $x, \varsigma \in\left\{x_{1}, x_{2}\right\}$. Then we have

$$
\begin{aligned}
& \mathbb{E}\left[\left|U\left(t, x_{1}\right)-U\left(t, x_{2}\right)\right|^{p}\right] \\
& \leq C\left|x_{1}-x_{2}\right|^{\theta p}\left[\int_{0}^{t}(t-s)^{-\theta}\left(F\left(x_{1}, x_{1}\right)+F\left(x_{1}, x_{2}\right)+F\left(x_{2}, x_{1}\right)+F\left(x_{2}, x_{2}\right)\right) d s\right]^{\frac{p}{2}} .
\end{aligned}
$$

When $x=\varsigma$, we use the Fourier's transform to obtain

$$
\begin{aligned}
F(x, x) & =\int_{\mathbb{R}^{d}} \int_{\mathbb{R}^{d}} \Gamma_{k_{2} / 2}(t, \mathbf{0} ; s, y) f(y-z) \Gamma_{k_{2} / 2}(t, \mathbf{0} ; s, z) d y d z \\
& =\int_{\mathbb{R}^{d}} \exp \left(-\frac{4 \pi^{2}(t-s)|\xi|^{2}}{k_{2}}\right) \mu_{f}(d \xi),
\end{aligned}
$$

where $\mathbf{0}$ is the origin in $d$-dimensional space. Applying the fact that (recalling $\eta \in(0,1)$ )

$$
\sup _{(t-s)|\xi|^{2} \in \mathbb{R}_{+}}\left\{\left(T+(t-s)|\xi|^{2}\right)^{1-\eta} \exp \left(-\frac{4 \pi^{2}(t-s)|\xi|^{2}}{k_{2}}\right)\right\}<\infty,
$$

we have

$$
F(x, x) \leq C(t-s)^{\eta-1} \int_{\mathbb{R}^{d}}\left(1+|\xi|^{2}\right)^{\eta-1} \mu_{f}(d \xi) \leq C(t-s)^{\eta-1}
$$


where $C$ is a constant independent of $x$. For the case $x \neq \varsigma$, we apply the definition of the non-negative tempered measure $\mu_{f}$ to obtain that

$$
\begin{aligned}
F(x, \varsigma) & =\int_{\mathbb{R}^{d}} \int_{\mathbb{R}^{d}} \Gamma_{k_{2} / 2}(t, \mathbf{0} ; s, y+\varsigma-x-(-z)) f(y) \Gamma_{k_{2} / 2}(t, \mathbf{0} ; s,-z) d y d z \\
& =\int_{\mathbb{R}^{d}} f(y)\left(\Gamma_{k_{2} / 2}(t, \mathbf{0} ; s, \cdot) * \Gamma_{k_{2} / 2}(t, \mathbf{0} ; s, \cdot)\right)(y+\varsigma-x) d y \\
& =\int_{\mathbb{R}^{d}} \exp (\xi \cdot(\varsigma-x) 2 \pi i) \exp \left(-\frac{4 \pi^{2}(t-s)|\xi|^{2}}{k_{2}}\right) \mu_{f}(d \xi) .
\end{aligned}
$$

Note that the integration is a real number, we have

$$
\int_{\mathbb{R}^{d}} \sin (2 \pi \xi \cdot(\varsigma-x)) \exp \left(-\frac{4 \pi^{2}(t-s)|\xi|^{2}}{k_{2}}\right) \mu_{f}(d \xi)=0,
$$

then we apply (3.5) and the nonnegativity of $\mu_{f}$ to get that

$$
F(x, \varsigma) \leq \int_{\mathbb{R}^{d}} \exp \left(-\frac{4 \pi^{2}(t-s)|\xi|^{2}}{k_{2}}\right) \mu_{f}(d \xi) \leq C(t-s)^{\eta-1},
$$

where $C$ is a constant independent of $t, s, x$, and $\varsigma$. Combine these estimates with (3.4), one has

$$
\mathbb{E}\left[\left|U\left(t, x_{1}\right)-U\left(t, x_{2}\right)\right|^{p}\right] \leq C\left|x_{1}-x_{2}\right|^{\theta p}\left(\int_{0}^{t}(t-s)^{\eta-\theta-1} d s\right)^{\frac{p}{2}} .
$$

To make the integral in the right side of (3.6) finite, we need $\theta \in(0, \eta)$. For any $\varepsilon>0$ small enough, we take $\theta=\eta-\varepsilon$. Then we have

$$
\mathbb{E}\left[\left|U\left(t, x_{1}\right)-U\left(t, x_{2}\right)\right|^{p}\right] \leq C\left|x_{1}-x_{2}\right|^{(\eta-\varepsilon) p},
$$

where $C$ is a constant independent of $t, x_{1}$, and $x_{2}$. Applying Lemma 3.2 to obtain that for any $\varepsilon>0$, the function $U$ has a modification, of which the trajectories are Hölder continuous in $x \in D$ with the exponent $\eta-\varepsilon$.

Step 2: To prove the continuity of $U$ in $t$, we set $0 \leq t_{2}<t_{1} \leq T$. Following the proof in Step 1, we have that for almost all $x \in \bar{D}$,

$$
\begin{aligned}
\mathbb{E}\left[\left|U\left(t_{1}, x\right)-U\left(t_{2}, x\right)\right|^{p}\right] \leq & C \mathbb{E}\left(\int_{t_{2}}^{t_{1}} \int_{D} \int_{D} G\left(t_{1}, x ; s, y\right) \cdot|\sigma(s, y, u(s, y))|\right. \\
& \left.\cdot f(y-z) \cdot|\sigma(s, z, u(s, z))| \cdot G\left(t_{1}, x ; s, z\right) d y d z d s\right)^{\frac{p}{2}} \\
+ & C \mathbb{E}\left(\int_{0}^{t_{2}} \int_{D} \int_{D}\left|G\left(t_{1}, x ; s, y\right)-G\left(t_{2}, x ; s, y\right)\right| \cdot|\sigma(s, y, u(s, y))|\right. \\
& \left.\cdot f(y-z)|\sigma(s, z, u(s, z))| \cdot\left|G\left(t_{1}, x ; s, z\right)-G\left(t_{2}, x ; s, z\right)\right| d y d z d s\right)^{\frac{p}{2}} \\
= & : I_{1}+I_{2} .
\end{aligned}
$$

Gaussian estimate (2.7), Assumption 2.5 and 2.6 yield

$$
\begin{aligned}
I_{1} & \leq\left(\int_{t_{2}}^{t_{1}} \int_{\mathbb{R}^{d}} \int_{\mathbb{R}^{d}} \Gamma_{k_{2}}\left(t_{1}, x ; s, y\right) f(y-z) \Gamma_{k_{2}}\left(t_{1}, x ; s, z\right) d y d z d s\right)^{\frac{p}{2}} \\
& \leq C\left(\int_{t_{2}}^{t_{1}}\left(t_{1}-s\right)^{\eta-1} d s\right)^{\frac{p}{2}} \leq C\left(t_{1}-t_{2}\right)^{\frac{\eta p}{2}}
\end{aligned}
$$


On the other hand, for any $\tau, t \in\left\{t_{1}, t_{2}\right\}$, define

$$
B(\tau, t):=\int_{\mathbb{R}^{d}} \int_{\mathbb{R}^{d}} \Gamma_{k_{2}}(\tau, x ; s, y) f(y-z) \Gamma_{k_{2}}(t, x ; s, z) d y d z,
$$

Set $\theta \in(0,1)$ a constant to be determined, then (3.2) indicates

$$
\begin{aligned}
I_{2} \leq & C\left(t_{1}-t_{2}\right)^{\frac{\theta p}{2}}\left[\int_{0}^{t_{2}}\left(t_{2}-s\right)^{-\theta} \int_{\mathbb{R}^{d}} \int_{\mathbb{R}^{d}}\left(\Gamma_{k_{2}}\left(t_{1}, x ; s, y\right)+\Gamma_{k_{2}}\left(t_{2}, x ; s, y\right)\right)\right. \\
& \left.\cdot f(y-z) \cdot\left(\Gamma_{k_{2}}\left(t_{1}, x ; s, z\right)+\Gamma_{k_{2}}\left(t_{2}, x ; s, z\right)\right) d y d z d s\right]^{\frac{p}{2}} \\
= & C\left(t_{1}-t_{2}\right)^{\frac{\theta p}{2}}\left[\int_{0}^{t_{2}}\left(t_{2}-s\right)^{-\theta}\left(B\left(t_{1}, t_{1}\right)+B\left(t_{1}, t_{2}\right)+B\left(t_{2}, t_{1}\right)+B\left(t_{2}, t_{2}\right)\right) d s\right]^{\frac{p}{2}} .
\end{aligned}
$$

When $\tau=t$, we apply (1.5) and (3.5) to obtain

$$
\begin{aligned}
B(\tau, t) & =\int_{\mathbb{R}^{d}} \int_{\mathbb{R}^{d}} \Gamma_{k_{2}}(\tau, x ; s, y) f(y-z) \Gamma_{k_{2}}(\tau, x ; s, z) d y d z \\
& =\int_{\mathbb{R}^{d}} \exp \left(-\frac{4 \pi^{2}(\tau-s)|\xi|^{2}}{k_{2}}\right) \mu_{f}(d \xi) \leq C(\tau-s)^{\eta-1},
\end{aligned}
$$

where the constant $C$ is independent of $\tau, s$, and $x$. For the case $\tau \neq t$, we also have

$$
\begin{aligned}
B(\tau, t) & =\int_{\mathbb{R}^{d}} \int_{\mathbb{R}^{d}} \Gamma_{k_{2}}(\tau, x ; s, y) f(y-z) \Gamma_{k_{2}}(t, x ; s, z) d y d z \\
& =\int_{\mathbb{R}^{d}} \exp \left(-\frac{2 \pi^{2}(\tau-s)|\xi|^{2}}{k_{2}}\right) \exp \left(-\frac{2 \pi^{2}(t-s)|\xi|^{2}}{k_{2}}\right) \mu_{f}(d \xi) \\
& \leq C \max \left\{(\tau-s)^{\eta-1},(t-s)^{\eta-1}\right\} .
\end{aligned}
$$

where $C$ is a constant independent of $\tau, s$, and $x$. Therefore, the estimate (3.7) shows

$$
I_{2} \leq C\left(t_{1}-t_{2}\right)^{\frac{\theta p}{2}}\left(\int_{0}^{t_{2}}\left(t_{2}-s\right)^{\eta-\theta-1} d s\right)^{\frac{p}{2}},
$$

where $C$ is a constant independent of $t_{1}, t_{2}$, and $x$. For any $\varepsilon>0$ small enough, we take the constant $\theta=\eta-2 \varepsilon$. Similar to the proof in Step 1 , we get that for any $\varepsilon>0$, the function $U$ has a modification, of which the trajectories are Hölder continuous in $t \in[0, T]$ with the exponent $\eta / 2-\varepsilon$.

For the joint space-time Hölder regularity, note that the following estimate

$$
\begin{aligned}
\mathbb{E}\left[\left|u\left(t_{1}, x_{1}\right)-u\left(t_{2}, x_{2}\right)\right|^{p}\right] & \leq C \mathbb{E}\left[\left|u\left(t_{1}, x_{1}\right)-u\left(t_{1}, x_{2}\right)\right|^{p}\right]+C \mathbb{E}\left[\left|u\left(t_{1}, x_{2}\right)-u\left(t_{2}, x_{2}\right)\right|^{p}\right] \\
& \leq C\left(\left|x_{1}-x_{2}\right|^{\eta p-\varepsilon}+\left|t_{1}-t_{2}\right|^{\eta p / 2-\varepsilon}\right)
\end{aligned}
$$

holds for all $t_{1}, t_{2} \in[0, T], x_{1}, x_{2} \in \bar{D}$, and $\varepsilon>0$ small enough, where $C$ is a constant independent of $t_{1}, t_{2}, x_{1}$, and $x_{2}$. Then we can apply Lemma 3.2 to complete the proof.

We conclude this paper with the proof of Lemma 3.1.

Proof of Lemma 3.1. For any $x, z \in \bar{D}$, define the infimum length of paths in $\bar{P}_{x, z}$ as $d_{D}(x, z)$. Obviously, for a fixed bounded domain $D$ with $C^{1+\beta}$ boundary, the function $d_{D}(x, z)$ is uniformly bounded (the upper bound is independent of the choice of points $x$ and $z$ ). Moreover, there exists a continuous path with the endpoints $x$ and $z$, which satisfies that each point on path is in $\bar{D}$, and the length of this path is $d_{D}(x, z)$. 
Suppose the constant $K_{D}$ does not exist, then there exist two sequences of points $\left\{x_{n}\right\}_{n \in \mathbb{N}}$ and $\left\{z_{n}\right\}_{n \in \mathbb{N}}$ such that $x_{n}, z_{n} \in \bar{D}$ for all $n \in \mathbb{N}$ and

$$
\lim _{n \rightarrow \infty} \frac{d_{D}\left(x_{n}, z_{n}\right)}{\left|x_{n}-z_{n}\right|}=\infty,
$$

which gives $\left|x_{n}-z_{n}\right| \stackrel{n \rightarrow \infty}{\longrightarrow} 0$. Since the domain $D$ is bounded, we can take the subsequences of $\left\{x_{n}\right\}_{n \in \mathbb{N}}$ and $\left\{z_{n}\right\}_{n \in \mathbb{N}}$, which also denote as $\left\{x_{n}\right\}_{n \in \mathbb{N}}$ and $\left\{z_{n}\right\}_{n \in \mathbb{N}}$ for convenience, such that $x_{n}$ and $z_{n}$ converge to the same point $e$ as $n \rightarrow \infty$. When $e$ in the interior of $D$, there exists a constant $\rho_{1}>0$ such that $B_{\rho_{1}}(e) \subset D$. Take $N$ large enough such that $x_{n}, z_{n} \in B_{\rho_{1}}(e)$ for any $n>N$. Then it is easy to find $d_{D}\left(x_{n}, z_{n}\right)=\left|x_{n}-z_{n}\right|$, which is contrary to (3.8).

For the case that $e \in \partial D$, define $\rho_{0}$ as the constant in Definition 2.1. Based on Definition 2.1, we can find a local coordinate system $\left(y^{1}, \ldots, y^{d}\right)$ such that the intersection of $B_{\rho_{0}}(e)$ with $\partial D$ has the form $y^{1}=\Psi\left(y^{2}, \ldots, y^{d}\right)$. Without loss of generality, we assume that the origin of local coordinate system is $e$, and the set $\left\{y^{1} \in B_{\rho_{0}}(e) \mid y^{1} \leq \Psi\left(y^{2}, \ldots, y^{d}\right)\right\}$ is in $\bar{D}$ (otherwise we use a new local coordinate system $\left(\bar{y}^{1}, \ldots, \bar{y}^{d}\right)$ and a new function $\bar{\Psi}$ such that $\bar{y}^{1}=-y^{1}, \bar{\Psi}=-\Psi$, and $\bar{y}^{i}=y^{i}$ for $\left.i=2, \ldots, d\right)$. Take $N$ large enough such that $x_{n}, z_{n} \in B_{\rho_{0}}(e)$ for all $n>N$. Now we fix $n>N$, and only consider the situation that the line segment $x_{n} z_{n}$ has the intersection with $\partial D\left(x_{n} z_{n}\right.$ denotes the line segment with the endpoints $x$ and $z$ ). Define the points $\bar{x}_{n}$ and $\bar{z}_{n}$ in the set $x_{n} z_{n} \cap \partial D$ as the closest points to $x$ and $z$, respectively. Then the line segment $\bar{x}_{n} \bar{z}_{n}$ has the representation

$$
\bar{y}_{n}(s)=\left(\bar{y}_{n}^{1}(s), \bar{y}_{n}^{2}(s), \ldots, \bar{y}_{n}^{d}(s)\right):=\bar{x}_{n}+s\left(\bar{z}_{n}-\bar{x}_{n}\right), \quad s \in[0,1]
$$

in the local coordinate system. Define a curve segment

$$
C_{\bar{x}_{n} \bar{z}_{n}}:=\left(\Psi\left(\bar{y}_{n}^{2}(s), \ldots, \bar{y}_{n}^{d}(s)\right), \bar{y}_{n}^{2}(s), \ldots, \bar{y}_{n}^{d}(s)\right), \quad s \in[0,1]
$$

then the curve segment $C_{\bar{x}_{n} \bar{z}_{n}}$ has the following properties:

(i) each point of $C_{\bar{x}_{n} \bar{z}_{n}}$ is on the boundary $\partial D$;

(ii) the endpoints of $C_{\bar{x}_{n} \bar{z}_{n}}$ are $\bar{x}_{n}$ and $\bar{z}_{n}$;

(iii) the projection of $C_{\bar{x}_{n} \bar{z}_{n}}$ on the surface $y^{1}=0$ is the same as the one of $\bar{x}_{n} \bar{z}_{n}$.

We claim that for a fixed point $e$, there exists a constant $K_{1}>1$, which is independent of $n$, such that the length of $C_{\bar{x}_{n} \bar{z}_{n}}$ is bounded by $K_{1}\left|\bar{x}_{n}-\bar{z}_{n}\right|$. This fact can be proved by the definition of integrating along curves and the boundedness of the first derivatives of

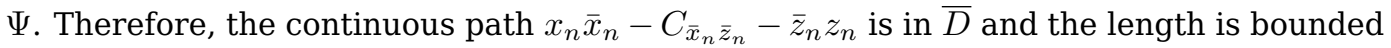
by $K_{1}\left|x_{n}-z_{n}\right|$. This property contradicts to (3.8), which proves Lemma 3.1.

\section{References}

[1] Le Chen and Jingyu Huang, Comparison principle for stochastic heat equation on $\mathbb{R}^{d}$, Ann. Probab. 47 (2019), no. 2, 989-1035. MR3916940

[2] Le Chen, Jingyu Huang, Davar Khoshnevisan, and Kunwoo Kim, Dense blowup for parabolic SPDEs, Electron. J. Probab. 24 (2019), Paper No. 118, 33. MR4029421

[3] Giuseppe Da Prato and Jerzy Zabczyk, Stochastic equations in infinite dimensions, second ed., Encyclopedia of Mathematics and its Applications, vol. 152, Cambridge University Press, Cambridge, 2014. MR3236753

[4] Robert C. Dalang, Extending the martingale measure stochastic integral with applications to spatially homogeneous s.p.d.e.'s, Electron. J. Probab. 4 (1999), no. 6, 29. MR1684157

[5] Robert C. Dalang and N. E. Frangos, The stochastic wave equation in two spatial dimensions, Ann. Probab. 26 (1998), no. 1, 187-212. MR1617046 
[6] Robert C. Dalang, Davar Khoshnevisan, Carl Mueller, David Nualart, and Yimin Xiao, A minicourse on stochastic partial differential equations, Lecture Notes in Mathematics, vol. 1962, Springer-Verlag, Berlin, 2009, Held at the University of Utah, Salt Lake City, UT, May 8-19, 2006, Edited by Khoshnevisan and Firas Rassoul-Agha. MR1500166

[7] Mohammud Foondun and Eulalia Nualart, On the behaviour of stochastic heat equations on bounded domains, ALEA Lat. Am. J. Probab. Math. Stat. 12 (2015), no. 2, 551-571. MR3382572

[8] N. Franzova, Long time existence for the heat equation with a spatially correlated noise term, Stochastic Anal. Appl. 17 (1999), no. 2, 169-190. MR1679747

[9] Avner Friedman, Partial differential equations of parabolic type, Prentice-Hall, Inc., Englewood Cliffs, N.J., 1964. MR0181836

[10] I. Gyöngy and É. Pardoux, On quasi-linear stochastic partial differential equations, Probab. Theory Related Fields 94 (1993), no. 4, 413-425. MR1201552

[11] István Gyöngy and É. Pardoux, On the regularization effect of space-time white noise on quasi-linear parabolic partial differential equations, Probab. Theory Related Fields 97 (1993), no. 1-2, 211-229. MR1240724

[12] István Gyöngy and Carles Rovira, On $L^{p}$-solutions of semilinear stochastic partial differential equations, Stochastic Process. Appl. 90 (2000), no. 1, 83-108. MR1787126

[13] Anna Karczewska and Jerzy Zabczyk, Stochastic PDE's with function-valued solutions, Infinite dimensional stochastic analysis (Amsterdam, 1999), Verh. Afd. Natuurkd. 1. Reeks. K. Ned. Akad. Wet., vol. 52, R. Neth. Acad. Arts Sci., Amsterdam, 2000, pp. 197-216. MR1832378

[14] Kyeong-Hun Kim, On stochastic partial differential equations with variable coefficients in $C^{1}$ domains, Stochastic Process. Appl. 112 (2004), no. 2, 261-283. MR2073414

[15] Kyeong-Hun Kim and N. V. Krylov, On the Sobolev space theory of parabolic and elliptic equations in $C^{1}$ domains, SIAM J. Math. Anal. 36 (2004), no. 2, 618-642. MR2111792

[16] N. V. Krylov, An analytic approach to SPDEs, Stochastic partial differential equations: six perspectives, Math. Surveys Monogr., vol. 64, Amer. Math. Soc., Providence, RI, 1999, pp. 185-242. MR1661766

[17] Hiroshi Kunita, Stochastic flows and stochastic differential equations, Cambridge Studies in Advanced Mathematics, vol. 24, Cambridge University Press, Cambridge, 1997, Reprint of the 1990 original. MR1472487

[18] O. A. Ladyženskaja, V. A. Solonnikov, and N. N. Ural'ceva, Linear and quasilinear equations of parabolic type, Translations of Mathematical Monographs, Vol. 23, American Mathematical Society, Providence, R.I., 1968, Translated from the Russian by S. Smith. MR0241822

[19] David Márquez-Carreras and Mònica Sarrà, Large deviation principle for a stochastic heat equation with spatially correlated noise, Electron. J. Probab. 8 (2003), no. 12, 39. MR1998765

[20] David Nualart and Lluís Quer-Sardanyons, Existence and smoothness of the density for spatially homogeneous SPDEs, Potential Anal. 27 (2007), no. 3, 281-299. MR2336301

[21] Eulalia Nualart, Moment bounds for some fractional stochastic heat equations on the ball, Electron. Commun. Probab. 23 (2018), Paper No. 41, 12. MR3841402

[22] Eulalia Nualart and Lluís Quer-Sardanyons, Gaussian estimates for the density of the nonlinear stochastic heat equation in any space dimension, Stochastic Process. Appl. 122 (2012), no. 1, 418-447. MR2860455

[23] M. Sanz-Solé and Mònica Sarrà, Hölder continuity for the stochastic heat equation with spatially correlated noise, Seminar on Stochastic Analysis, Random Fields and Applications, III (Ascona, 1999), Progr. Probab., vol. 52, Birkhäuser, Basel, 2002, pp. 259-268. MR1958822

[24] Marta Sanz-Solé and Mònica Sarrà, Path properties of a class of Gaussian processes with applications to spde's, Stochastic processes, physics and geometry: new interplays, I (Leipzig, 1999), CMS Conf. Proc., vol. 28, Amer. Math. Soc., Providence, RI, 2000, pp. 303-316. MR1803395

[25] Marta Sanz-Solé and Pierre-A. Vuillermot, Equivalence and Hölder-Sobolev regularity of solutions for a class of non-autonomous stochastic partial differential equations, Ann. Inst. H. Poincaré Probab. Statist. 39 (2003), no. 4, 703-742. MR1983176 
The Dirichlet problem for SPDEs

[26] Shijie Shang and Ran Wang, Transportation inequalities under uniform metric for a stochastic heat equation driven by time-white and space-colored noise, Acta Appl. Math. 170 (2020), 81-97. MR4163229

[27] John B. Walsh, An introduction to stochastic partial differential equations, École d'été de probabilités de Saint-Flour, XIV-1984, Lecture Notes in Math., vol. 1180, Springer, Berlin, 1986, pp. 265-439. MR0876085 


\section{Electronic Journal of Probability Electronic Communications in Probability}

\section{Advantages of publishing in EJP-ECP}

- Very high standards

- Free for authors, free for readers

- Quick publication (no backlog)

- Secure publication $\left(\mathrm{LOCKSS}^{1}\right)$

- Easy interface (EJMS²)

\section{Economical model of EJP-ECP}

- Non profit, sponsored by $\mathrm{IMS}^{3}, \mathrm{BS}^{4}$, ProjectEuclid ${ }^{5}$

- Purely electronic

\section{Help keep the journal free and vigorous}

- Donate to the IMS open access fund ${ }^{6}$ (click here to donate!)

- Submit your best articles to EJP-ECP

- Choose EJP-ECP over for-profit journals

\footnotetext{
${ }^{1}$ LOCKSS: Lots of Copies Keep Stuff Safe http://www. lockss.org/

${ }^{2}$ EJMS: Electronic Journal Management System http://www.vtex.lt/en/ejms.html

${ }^{3}$ IMS: Institute of Mathematical Statistics http://www.imstat.org/

${ }^{4}$ BS: Bernoulli Society http://www. bernoulli-society.org/

${ }^{5}$ Project Euclid: https://projecteuclid.org/

${ }^{6}$ IMS Open Access Fund: http://www.imstat.org/publications/open.htm
} 